\section{(2) OPEN ACCESS}

\title{
Clinical determinants of plasma cardiac biomarkers in patients with stable chest pain
}

\author{
Rong Bing (D) ,' James Henderson, ${ }^{1}$ Amanda Hunter, ${ }^{1}$ Michelle C Williams (D) , 1,2 \\ Alastair J Moss, ${ }^{1}$ Anoop S V Shah, ${ }^{1}$ David A McAllister (1) ,' Marc R Dweck, ${ }^{1,2}$ \\ David E Newby, ${ }^{1,2}$ Nicholas L Mills (D) , 1,4 Philip D Adamson (1) 1,5
}

\begin{abstract}
- Additional material is published online only. To view please visit the journal online (http://dx.doi.org/10.1136/ heartjnl-2019-314892).
\end{abstract}

${ }^{1} \mathrm{BHF}$ Centre for Cardiovascular Science, University of Edinburgh, Edinburgh, UK

${ }^{2}$ Edinburgh Imaging, University of Edinburgh, Edinburgh, UK

${ }^{3}$ Institute of Health and Wellbeing, University of Glasgow, Glasgow, UK ${ }^{4}$ Usher Institute of Population Health Sciences and Informatics, University of Edinburgh, Edinburgh, UK

${ }^{5}$ Christchurch Heart Institute, University of Otago,

Christchurch, New Zealand

\section{Correspondence to} Dr Rong Bing, Centre for Cardiovascular Science, University of Edinburgh, Edinburgh EH8 9YL, UK rongbing.rb@gmail.com

Received 12 February 2019 Revised 8 May 2019 Accepted 10 May 2019 Published Online First 1 June 2019
ABSTRACT
Objective

Objective Troponin and B-type natriuretic peptide (BNP) concentrations are associated with cardiovascular risk in stable patients. Understanding their determinants and identifying modifiable clinical targets may improve outcomes. We aimed to establish clinical and cardiac determinants of these biomarkers.

Methods This was a prespecified substudy from the randomised Scottish Computed Tomography of the Heart trial, which enrolled patients $18-75$ years with suspected stable angina between 2010 and 2014 (NCT01149590). We included patients from six centres in whom highsensitivity troponin I and BNP were measured (Singulex Erenna). Patients with troponin $>99$ th centile upper reference limit $(10.2 \mathrm{ng} / \mathrm{L})$ or $B N P \geq 400 \mathrm{ng} / \mathrm{L}$ were excluded to avoid inclusion of patients with myocardial injury or heart failure. Multivariable linear regression models were constructed with troponin and BNP as dependent variables.

Results In total, 885 patients were included; 881 (99\%) and 847 (96\%) had troponin and BNP concentrations above the limit of detection, respectively. Participants had a slight male preponderance $(n=513 ; 56.1 \%)$, and the median age was 59.0 (IQR 51.0-65.0) years. The median troponin and BNP concentrations were 1.4 (IQR 0.90-2.1) ng/L and 29.1 (IQR 14.0-54.0) ng/L, respectively. Age and atherosclerotic burden were independent predictors of both biomarkers. Male sex, left ventricular mass and systolic blood pressure were independent predictors of increased troponin. In contrast female sex and left ventricular volume were independent predictors of increased BNP.

Conclusions Troponin and BNP are associated with coronary atherosclerosis but have important sex differences and distinct and contrasting associations with CT-determined left ventricular mass and volume. Clinical Trial registration NCT01149590; Postresults.

\section{INTRODUCTION}

The diagnostic and prognostic application of myocardial-specific plasma proteins is widely accepted in current practice. Two such cardiovascular biomarkers are high-sensitivity cardiac troponin and B-type natriuretic peptide (BNP). Although developed for use in specific populations, both are recognised to be important indicators of adverse prognosis among stable patients, even in the absence of established cardiac disease. ${ }^{1-4}$
These associations likely reflect the role of myocardial proteins as surrogate measures of underlying processes such as hypertension, atherosclerosis or left ventricular dysfunction. ${ }^{5}{ }^{6}$ Indeed, there have been suggestions that measurement of high-sensitivity troponin and BNP in certain asymptomatic populations may be of benefit. ${ }^{7}$ However, these tests are not specific for a single disease process. Understanding the drivers of biomarker concentrations in an individual patient is therefore crucial to facilitate interpretation of results and guide management.

Cardiac CT is a valuable investigation that is widely used for the diagnosis of coronary artery disease. However, it can also provide an assessment of left ventricular mass and volume, both of which are associated with elevated biomarker concentrations. ${ }^{89}$ How these measures contribute to variations in biomarker concentrations in stable patients remains unclear.

In this biomarker substudy of the Scottish Computed Tomography of the Heart (SCOTHEART) trial, we aimed to establish the clinical and cardiac determinants of plasma high-sensitivity cardiac troponin I and BNP concentrations in patients presenting with stable chest pain. We hypothesised that, in addition to recognised clinical determinants, ventricular mass and volume as determined by cardiac CT would be associated with higher biomarker concentrations.

\section{METHODS}

\section{Study design and population}

This is a post-hoc analysis of the open-label, randomised SCOT-HEART trial. The trial design, primary analysis and 5-year outcomes have been published. ${ }^{10-12}$ Patients $18-75$ years of age referred by a primary care physician to a cardiology clinic with stable chest pain were enrolled after obtaining written informed consent from 12 cardiology centres across Scotland. In total, 4146 patients were recruited from November 2010 to September 2014. Patients with severe chronic kidney disease (serum creatinine $>200 \mu \mathrm{mol} / \mathrm{L}$ or estimated glomerular filtration rate $<30 \mathrm{~mL} / \mathrm{min} / 1.73 \mathrm{~m}^{2}$ ) or acute coronary syndrome within 3 months were excluded. All patients underwent routine clinical evaluation. A clinical diagnosis and management plan were documented prior to recruitment. Eligible patients were randomised 1:1 to receive routine care or routine care plus CT coronary angiography (CTCA). 
For this substudy, we included patients who had been referred from six centres for their allocated CTCA in Edinburgh $(n=1317)$. As this study aimed to characterise the determinants of baseline cardiac biomarkers in stable patients without acute myocardial injury or heart failure, patients with troponin concentrations $>99$ th centile upper reference limit $(10.2 \mathrm{ng} / \mathrm{L})$ $(n=29)$ or BNP $\geq 400 \mathrm{ng} / \mathrm{L}(\mathrm{n}=11)^{13}$ were excluded.

\section{Computed tomography coronary angiography}

The methods used to perform and to analyse coronary findings from CTCA have been reported. ${ }^{12}$ Intraobserver and interobserver agreement were excellent $(95 \%$ and $91 \%$, respectively). ${ }^{10}$ Atherosclerotic burden was quantified according to the CT-adapted Leaman score. This validated tool provides a score based on plaque location, composition (non-calcified, calcified or mixed) and degree of stenosis $(<50 \%$ or $\geq 50 \%)$. The score has been validated in several cohorts; a cut-off of $>5$ provides additive prognostic value beyond stenosis alone. ${ }^{14}$

\section{Left ventricular mass and volume}

Cardiac CT assessments of myocardial mass and volume are validated and readily performed. Older protocols used retrospective electrocardiographic gating; however, prospective gating is now routine, with image acquisition performed in mid-diastole. Although this limits the available phases, mid-diastolic left ventricular mass measurements correlate well with other phases and have prognostic value. ${ }^{15}$ Left ventricular mid-diastolic volume measurements also correlate with end-diastolic volumes and have prognostic importance, ${ }^{16}$ with established thresholds for normal ranges. ${ }^{17} 18$ Due to variation in volume measurements across the cardiac cycle, we only included scans with gated images available between $70 \%$ and $90 \%$ of the R-R interval for this metric. In contrast, estimation of left ventricular mass is less affected by the cardiac cycle; we therefore included scans with images between $50 \%$ and $90 \%$ of the R-R interval.

Using automated software (Vitrea, Vital Images, Minnetonka, USA), left ventricular myocardial contours were detected during mid-diastole ( $75 \%$ phase or nearest acquired) in two planes and $0.7 \mathrm{~mm}$ slices and manually adjusted to ensure accurate borders, allowing calculation of left ventricular volume and myocardial volume (from which myocardial mass is derived). Corrections for mid-diastolic acquisition were then applied ${ }^{15} 19$ to provide mass and estimated left ventricular end-diastolic volume. Values were indexed to body surface area (Du Bois formula). Left ventricular hypertrophy was defined as indexed left ventricular mass $>79.2 \mathrm{~g} / \mathrm{m}^{2}$ for men and $>63.8 \mathrm{~g} / \mathrm{m}^{2}$ for women-CT-specific thresholds with prognostic value. ${ }^{15}$

\section{Biomarker measurement}

Troponin was measured using the Erenna high-sensitivity cardiac troponin I assay (Singulex, Alameda, California). The limit of detection is $0.1 \mathrm{ng} / \mathrm{L}$ with a 99 th centile upper reference limit of $10.2 \mathrm{ng} / \mathrm{L}$ and a limit of quantification (LOQ; coefficient of variation $<10 \%$ ) of $0.4 \mathrm{ng} / \mathrm{L}{ }^{20} 21$ The BNP assay (Singulex) had a limit of detection of $2.0 \mathrm{ng} / \mathrm{L}$ and LOQ (coefficient of variation $<10 \%$ ) of $3.4 \mathrm{ng} / \mathrm{L}$. EDTA-treated plasma samples were stored at $-80^{\circ} \mathrm{C}$.

\section{Statistical analysis}

Analyses were performed using biomarker concentrations as continuous variables following $\log _{2}$ transformation. For descriptive purposes baseline characteristics are reported as median (IQR) according to troponin and BNP quantiles. Body mass index, systolic blood pressure, diastolic blood pressure, CT-adapted Leaman score, indexed left ventricular mass, indexed left ventricular volume and creatinine were log-transformed as a linearising transformation. Missingness was low, apart from creatinine $(n=220,25 \%)$ and indexed left ventricular volume $(\mathrm{n}=195,22 \%$; due to available scan phases and image quality). To avoid introducing bias by using complete case analysis, missing values were estimated by multivariable imputation by chained equations. Subsequent analyses were conducted using a single imputed value for each missing value. In a sensitivity analysis, the pooled parameters for the final models were also determined (online supplementary appendix). ${ }^{22}$ Patients with troponin or BNP concentrations below the lower limit of detection ( $n=4$ and $n=38$, respectively) were assigned a value of half the lower limit of detection. Univariable and multivariable linear regression modelling were performed to identify the associations between each biomarker and relevant clinical, biochemical and imaging-derived variables. Included variables were CT-adapted Leaman score, left ventricular mass and volume indexed to body surface area, age (per decade), male sex, hypertension, hyperlipidaemia, diabetes, family history of coronary heart disease, documented coronary heart disease, smoking history, systolic blood pressure, diastolic blood pressure, body mass index, and serum creatinine. Sequential multivariable models were built, beginning with CT-adapted Leaman score and indexed left ventricular mass and volume, followed by the addition of clinical history, clinical measurements and biochemistry (online supplementary tables 1 and 2). Two-sided $\mathrm{p}$ values $<0.05$ were considered statistically significant. Analysis was performed using R V.3.5.0 (R Foundation for Statistical Computing, Vienna, Austria).

\section{RESULTS}

\section{Patient characteristics}

In total, 943 patients underwent biomarker sampling, of whom $29(3 \%)$ had a troponin concentration $>99$ th centile upper reference limit and $11(1 \%)$ had a BNP $>400 \mathrm{ng} / \mathrm{L}$. After excluding these patients and patients with missing BNP data, 885 patients were included. The median troponin concentration was 1.4 (0.9-2.1) ng/L. The median BNP concentration was 29.1 (14.0$54.0) \mathrm{ng} / \mathrm{L}$ (figure 1). Patients with higher biomarker concentrations were older, more likely to have a history of coronary heart disease, be receiving medical therapies for cardiovascular conditions and have a typical history of angina (table 1). Troponin concentrations were higher in men than in women (1.6 (1.12.1) vs $1.0(0.7-1.6) \mathrm{ng} / \mathrm{L})$ in contrast to $\mathrm{BNP}$ concentrations which were higher in women than in men (31.9 (17.7-58.1) vs $26.5(12.5-50.0) \mathrm{ng} / \mathrm{L})$. There was a weak correlation between troponin and BNP concentrations (Pearson's $r=0.21, \mathrm{p}<0.001$ ).

\section{Coronary artery disease}

Coronary CT angiography demonstrated normal coronary arteries in 309 (35\%) patients, while $401(45 \%)$ patients had stenoses of at least $50 \%$ (table 1 ). The median calcium score was 30 (0.00-269.0) Agatston units. The median CT-adapted Leaman score was $4.0(0.00-10.9)$, and $406(46 \%)$ patients had high-risk coronary artery disease (CT-adapted Leaman score $>5$ ). In 576 patients with evidence of any coronary artery disease on CT, the median calcium score was 143.5 (31.0-530.5) Agatston units, while the median CT-adapted Leaman score was 8.3 (4.4-14.2).

\section{Left ventricular mass and volume}

The median indexed left ventricular mass was $78.1(71.1-86.1) \mathrm{g} /$ $\mathrm{m}^{2}$ for men and $62.6(56.7-69.9) \mathrm{g} / \mathrm{m}^{2}$ for women. Nearly half 


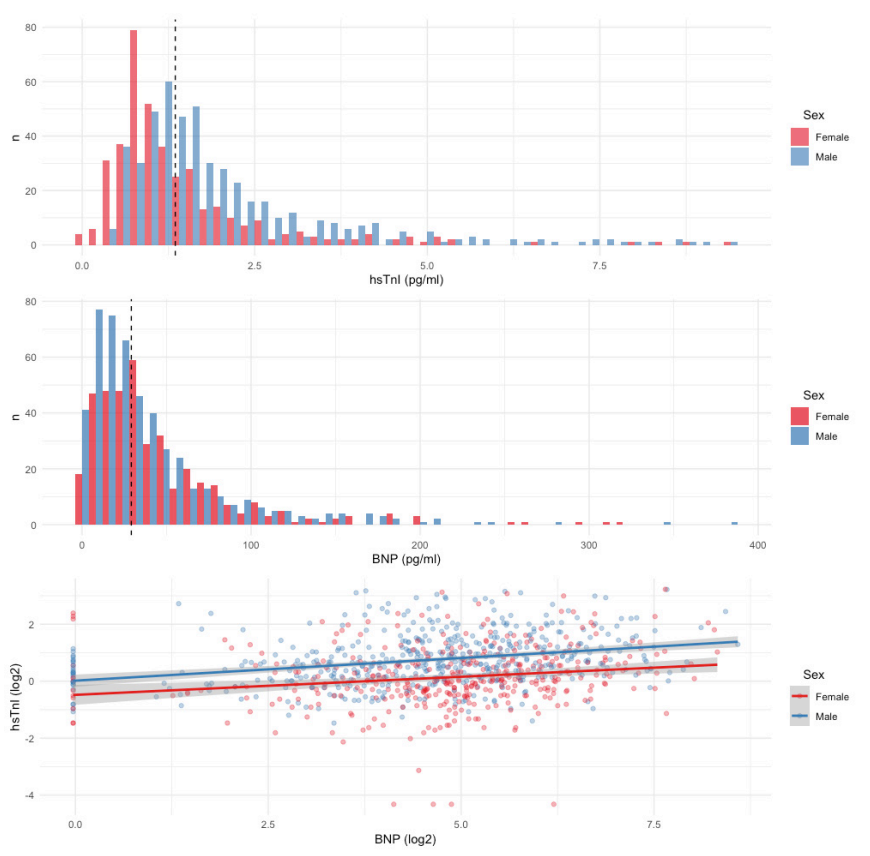

Figure 1 Distribution of cardiac biomarkers between men and women. Panels A and B demonstrate the distribution of high-sensitivity troponin I (hsTnl) and B-type natriuretic peptide (BNP), stratified by gender. Dashed lines represent median values for hsTnl (1.4 ng/L) and BNP ( $29.1 \mathrm{ng} / \mathrm{L})$ for the whole cohort. Panel C represents univariable linear regression modelling that demonstrates the weak association between hsTnl and BNP for both women $\left(r^{2}=0.03\right.$, coefficient 0.13 , $p<0.001)$ and men $\left(r^{2}=0.09\right.$, coefficient $\left.0.16, p<0.001\right)$.

$(n=404,46 \%)$ of patients met sex-specific CT criteria for left ventricular hypertrophy. The median indexed left ventricular volume was $64.6(56.6-71.3) \mathrm{mL} / \mathrm{m}^{2}$ for men and $62.1(56.5-$ $68.0) \mathrm{mL} / \mathrm{m}^{2}$ for women.

\section{Determinants of plasma cardiac biomarkers}

Univariable and sequential multivariable linear regression were performed using the prespecified variables for troponin (table 2) and BNP (table 3). There was an association between troponin and BNP measurements on univariable linear regression for both men and women (figure 1). For the final multivariable models, all prespecified covariates for troponin $\left(\mathrm{r}^{2}=0.30\right.$, $\mathrm{p}<0.001)$ and $\mathrm{BNP}\left(\mathrm{r}^{2}=0.19, \mathrm{p}<0.001\right)$ were included. Increasing age and atherosclerotic burden as assessed by CT-adapted Leaman score were independent predictors for increased troponin and BNP concentrations. Male sex and indexed left ventricular mass were predictors of increased troponin, in addition to hypertension, systolic blood pressure and body mass index, but not BNP. In contrast, female sex and indexed left ventricular volume were predictors of increased BNP but not troponin. Of note, creatinine was a univariable predictor of increased troponin, but the association no longer remained after adjustment for other variables. Conversely, creatinine was a multivariable predictor of lower BNP. The biomarker-specific nature of these associations was confirmed on multivariable analysis of variance testing of the full model where both biomarkers were included as dependent variables (bivariate multivariable regression). Furthermore, after standardising continuous variables, indexed left ventricular mass and volume provided the largest relative contribution to the explained variance within the troponin and BNP multivariable models, respectively, apart from age, which demonstrated the highest coefficient for both models (figure 2). Finally, to assess the potential effect of data imputation, complete case analysis was performed $(n=495)$. Pooled model parameters were also determined (online supplementary tables 3 and 4). The multivariable models were largely unchanged. The direction and magnitude for the associations between age, gender, structural CT parameters, creatinine and both biomarkers were similar, although with expected variance in SEs.

\section{DISCUSSION}

In this analysis from the SCOT-HEART trial, we describe clinical determinants of plasma high-sensitivity cardiac troponin I and BNP concentrations in a cohort of patients with suspected stable angina. We demonstrate the ability of cardiac CT to identify and characterise three distinct determinants of these biomarker concentrations: atherosclerotic burden, left ventricular mass and left ventricular volume. This has particular clinical relevance as troponin and BNP are powerful predictors of cardiovascular risk. These quantifiable imaging measures offer potentially modifiable targets for intervention.

It is imperative that patient-specific mechanisms for biomarker concentrations are understood. Are they a marker of an active, modifiable disease process, or simply sentinels for generalised, systemic inflammation and elevated cardiovascular risk? Without this understanding, targeted interventions cannot be applied, thereby diminishing the clinical benefits of additional prognostic information. The importance of a mechanistic approach is demonstrated by the success of canakinumab, which specifically targets inflammation as a driver of cardiovascular events. ${ }^{23}$ Importantly, a number of therapies have already been demonstrated to address the processes we identified as contributing to increased biomarker concentrations. This provides a strong basis for using cardiac imaging when investigating the cause of these increased biomarker concentrations.

Our findings suggest that cardiac CT can achieve some of the necessary characterisation of biomarker determinants in stable patients. Obstructive coronary artery disease correlates with troponin concentrations in patients with stable angina. ${ }^{24}$ Here, we applied the CT-adapted Leaman score and demonstrate that, in addition to other clinical characteristics, this association persists after adjustment for left ventricular mass and volume. Although atherosclerotic disease is a chronic inflammatory process, changes within the vasculature are distinct from structural processes; cardiac CT facilitates the interrogation of both these aspects. Left ventricular mass measured at end-diastole using other modalities has been shown to correlate with elevations in cardiac biomarkers. ${ }^{25}$ Our study confirms a comparable relationship between troponin and mid-diastolic assessment of left ventricular mass on gated CT. Additionally, BNP concentrations are elevated in heart failure or conditions with increased wall stress leading to left ventricular hypertrophy. ${ }^{9}$ However, we have demonstrated that after adjustment, left ventricular volume remains an independent determinant of BNP in stable patients without evidence of heart failure, whereas mass does not. The ability to perform these measurements with prospective gating is highly relevant in current practice. Of note, although we were unable to compare mid-diastolic left ventricular mass or volume directly with echocardiography or cardiac MRI, excellent correlation with other cardiac phases has been demonstrated previously. ${ }^{15} 1718$

Our study is consistent with prior reports demonstrating an association between plasma biomarker concentrations and several clinical factors. Advanced age and male gender are both 
Table 1 Baseline characteristics for overall cohort and according to high-sensitivity cardiac troponin I and BNP quantiles (below/above median)

\begin{tabular}{|c|c|c|c|c|c|c|c|}
\hline & \multirow[b]{2}{*}{$\begin{array}{l}\text { Overall } \\
\mathrm{N}=885\end{array}$} & \multicolumn{3}{|l|}{ hs-cTnl } & \multicolumn{3}{|l|}{ BNP } \\
\hline & & $\begin{array}{l}\text { Low }(\leq 1.4 \mathrm{ng} / \mathrm{L}) \\
\mathrm{n}=443\end{array}$ & $\begin{array}{l}\operatorname{High}(>1.4 \mathrm{ng} / \mathrm{L}) \\
\mathrm{n}=442\end{array}$ & $P$ value & $\begin{array}{l}\text { Low }(\leq 29.1 \mathrm{ng} / \mathrm{L}) \\
\mathrm{n}=443\end{array}$ & $\begin{array}{l}\text { High (>29.1 ng/L) } \\
\mathrm{n}=442\end{array}$ & $P$ value \\
\hline Male & $494(55.8)$ & $192(43.3)$ & $302(68.3)$ & $<0.001$ & $267(60.3)$ & $227(51.4)$ & 0.009 \\
\hline Age & $59.0(51.0-65.0)$ & $55.0(48.0-63.0)$ & $62.0(55.0-67.0)$ & $<0.001$ & $55.0(48.0-62.0)$ & $62.0(55.0-67.0)$ & $<0.001$ \\
\hline Hypertension & $325(36.7)$ & $119(26.9)$ & $206(46.6)$ & $<0.001$ & $149(33.6)$ & $176(39.8)$ & 0.07 \\
\hline Hyperlipidaemia & $528(59.7)$ & $246(55.5)$ & $282(63.8)$ & 0.015 & $233(52.6)$ & $295(66.7)$ & $<0.001$ \\
\hline Diabetes & $91(10.3)$ & $41(9.3)$ & $50(11.3)$ & 0.37 & $50(11.3)$ & $41(9.3)$ & 0.38 \\
\hline Known CAD & $77(8.7)$ & $24(5.4)$ & $53(12.0)$ & 0.001 & $23(5.2)$ & $54(12.2)$ & $<0.001$ \\
\hline Family history of CAD & $383(43.3)$ & $214(48.3)$ & $169(38.2)$ & 0.003 & $199(44.9)$ & $184(41.6)$ & 0.36 \\
\hline \multicolumn{8}{|l|}{ Smoking history } \\
\hline Non-smoker & $396(44.7)$ & $192(43.3)$ & $204(46.2)$ & 0.009 & $203(45.8)$ & $193(43.7)$ & 0.07 \\
\hline Ex-smoker & $313(35.4)$ & $145(32.7)$ & $168(38.0)$ & & $142(32.1)$ & $171(38.7)$ & \\
\hline Current smoker & $176(19.9)$ & $106(23.9)$ & $70(15.8)$ & & $98(22.1)$ & $78(17.6)$ & \\
\hline Atrial fibrillation & $14(1.6)$ & $6(1.4)$ & $8(1.8)$ & 0.78 & $8(1.8)$ & $6(1.4)$ & 0.79 \\
\hline Cerebrovascular disease & $38(4.3)$ & $18(4.1)$ & $20(4.5)$ & 0.87 & $16(3.6)$ & $22(5.0)$ & 0.40 \\
\hline PVD & $17(1.9)$ & $8(1.8)$ & $9(2.0)$ & 1.00 & $7(1.6)$ & $10(2.3)$ & 0.63 \\
\hline \multicolumn{8}{|l|}{ Chest pain type } \\
\hline Non-anginal & $303(34.2)$ & $179(40.4)$ & $124(28.1)$ & $<0.001$ & $183(41.3)$ & $120(27.1)$ & $<0.001$ \\
\hline Atypical angina & $211(23.8)$ & $119(26.9)$ & $92(20.8)$ & & $108(24.4)$ & $103(23.3)$ & \\
\hline Typical angina & $371(41.9)$ & $145(32.7)$ & $226(51.1)$ & & $152(34.3)$ & $219(49.5)$ & \\
\hline Systolic blood pressure (mm Hg) & $140.0(128.0-154.0)$ & $138.0(124.0-151.0)$ & $140.0(130.0-156.8)$ & $<0.001$ & $140.0(128.0-152.0)$ & $140.0(128.0-155.0)$ & 0.30 \\
\hline $\begin{array}{l}\text { Diastolic blood pressure } \\
(\mathrm{mm} \mathrm{Hg})\end{array}$ & $81.0(76.0-90.0)$ & $80.0(75.0-90.0)$ & $82.0(76.0-90.0)$ & 0.04 & $82.0(77.0-90.0)$ & $80.0(75.0-90.0)$ & 0.12 \\
\hline $\mathrm{BMI}\left(\mathrm{kg} / \mathrm{m}^{2}\right)$ & $28.7(25.9-32.6)$ & $28.6(25.5-32.3)$ & $29.0(26.2-32.9)$ & 0.13 & $29.1(26.3-33.3)$ & $28.2(25.5-32.2)$ & 0.02 \\
\hline Antiplatelet & $448(50.6)$ & $189(42.7)$ & $259(58.6)$ & $<0.001$ & $183(41.3)$ & $265(60.0)$ & $<0.001$ \\
\hline Statin & $393(44.4)$ & $171(38.6)$ & $222(50.2)$ & 0.001 & $158(35.7)$ & $235(53.2)$ & $<0.001$ \\
\hline ACE inhibitor & $123(13.9)$ & $40(9.0)$ & $83(18.8)$ & $<0.001$ & $48(10.8)$ & $75(17.0)$ & 0.01 \\
\hline Beta-blocker & $202(22.8)$ & $84(19.0)$ & $118(26.7)$ & 0.008 & $72(16.3)$ & $130(29.4)$ & $<0.001$ \\
\hline ASSIGN score & $16.0(10.0-23.0)$ & $13.0(8.0-21.0)$ & $18.0(13.0-26.0)$ & $<0.001$ & $14.0(9.0-22.0)$ & $17.0(12.0-25.0)$ & $<0.001$ \\
\hline Creatinine $(\mu \mathrm{mol} / \mathrm{L})$ & $\begin{array}{l}91.1 \\
(76.7-109.5)\end{array}$ & $\begin{array}{l}88.6 \\
(74.4-106.3)\end{array}$ & $\begin{array}{l}93.5 \\
(79.6-111.7)\end{array}$ & $<0.001$ & $\begin{array}{l}91.4 \\
(77.4-111.4)\end{array}$ & $\begin{array}{l}90.6 \\
(76.5-108.2)\end{array}$ & 0.3 \\
\hline hs-cTnl (ng/L) & $1.3(0.9-2.1)$ & $0.9(0.7-1.1)$ & $2.1(1.6-3.2)$ & $<0.001$ & $1.2(0.8-1.8)$ & $1.5(1.0-2.4)$ & $<0.001$ \\
\hline $\mathrm{BNP}$ (ng/L) & $29.1(14.2-54.0)$ & $24.9(11.2-44.1)$ & $34.9(19.2-67.1)$ & $<0.001$ & $14.2(7.8-21.7)$ & $54.1(39.6-83.8)$ & $<0.001$ \\
\hline \multicolumn{8}{|l|}{ CAD } \\
\hline Normal & $309(34.9)$ & $204(46.0)$ & $105(23.8)$ & $<0.001$ & $178(40.2)$ & $131(29.6)$ & $<0.001$ \\
\hline Mild (<50\%) & $175(19.8)$ & $88(19.9)$ & $87(19.7)$ & & $94(21.2)$ & $81(18.3)$ & \\
\hline Moderate $(50 \%-70 \%)$ & $159(18.0)$ & 77 (17.4) & $82(18.6)$ & & $76(17.2)$ & $83(18.8)$ & \\
\hline Obstructive (>70\%) & $242(27.3)$ & $74(16.7)$ & $168(38.0)$ & & $95(21.4)$ & $147(33.3)$ & \\
\hline CT-adapted Leaman score & $4.0(0.0-10.9)$ & $1.5(0.0-6.9)$ & $7.0(0.9-14.4)$ & $<0.001$ & $3.1(0.0-8.7)$ & $5.5(0.0-12.2)$ & $<0.001$ \\
\hline Calcium score (Agatston units) & $30.0(0.0-269.0)$ & $3.0(0.0-95.0)$ & $103.5(1.2-546.5)$ & $<0.001$ & $11.0(0.0-136.5)$ & $63.5(0.0-430.2)$ & $<0.001$ \\
\hline $\begin{array}{l}\text { Indexed left ventricular volume } \\
\left(\mathrm{mL} / \mathrm{m}^{2}\right)\end{array}$ & $63.2(56.6-69.5)$ & $63.2(57.2-69.6)$ & $62.5(55.8-69.3)$ & 0.30 & $62.6(56.2-69.7)$ & $63.2(56.9-69.4)$ & 0.72 \\
\hline $\begin{array}{l}\text { Indexed left ventricular mass } \\
\left(\mathrm{g} / \mathrm{m}^{2}\right)\end{array}$ & $71.7(62.6-81.5)$ & $67.6(59.6-76.8)$ & $75.7(67.6-84.4)$ & $<0.001$ & $72.6(63.4-81.7)$ & $70.8(61.6-81.2)$ & 0.17 \\
\hline Left ventricular hypertrophy & $404(45.6)$ & $174(39.3)$ & $230(52.0)$ & $<0.001$ & $203(45.8)$ & $201(45.5)$ & 0.97 \\
\hline
\end{tabular}

Continuous variables are presented as median (IQR)

Categorical variables are presented as $\mathrm{n}(\%)$.

BMI, body mass index; BNP, B-type natriuretic peptide; CAD, coronary artery disease; CTCA, CT coronary angiography; hs-cTnl, high-sensitivity cardiac troponin l; PVD, peripheral vascular disease.

associated with increased troponin concentrations. ${ }^{6} 8$ There has been considerable research into determining age-specific and sex-specific 99th centile thresholds for each assay. Although posited explanations include a higher atherosclerotic burden in the elderly and increased myocardial mass in men, we have shown that age and male gender remain predictors of higher troponin concentrations even after adjusting for these variables. Likewise, we also describe the association between female sex and increased BNP which persisted after adjustment for confounders such as age, atherosclerotic burden and left ventricular mass and volume-variables that are not reported in previous studies. This demonstrates the utility of cardiac CT in delineating how coronary and structural parameters contribute to BNP elevation. ${ }^{3}$ The precise mechanisms for these independent gender differences are unclear. Hormonal differences are likely to contribute. Oestrogen has an attenuating effect on atherosclerosis and left ventricular hypertrophy, ${ }^{26}$ while testosterone suppresses natriuretic peptide production, resulting in lower concentrations. ${ }^{27}$ Although the mechanisms are uncertain, our findings support the application of sex-specific reference ranges, and furthermore point towards the need for age-specific cut-offs. Universal thresholds may misclassify patients, potentially leading to inappropriate therapies.

In contrast to prior reports that elevated troponin concentrations are more common in outpatients with chronic kidney disease, ${ }^{28} 29$ we found that a higher creatinine was not an independent predictor of increased troponin. In contrast, creatinine was associated with a lower BNP concentration after 
Table 2 Univariable and multivariable associations for high-sensitivity cardiac troponin I concentration

\begin{tabular}{|c|c|c|c|c|c|c|}
\hline & \multicolumn{3}{|c|}{ Univariable regression analysis } & \multicolumn{3}{|c|}{ Multivariable regression analysis ${ }^{*} r^{2}=0.30$} \\
\hline & Coefficient & $95 \% \mathrm{Cl}$ & $P$ value & Coefficient & $95 \% \mathrm{Cl}$ & $P$ value \\
\hline Age (per 10 years) & 0.37 & 0.30 to 0.44 & $<0.001$ & 0.33 & 0.25 to 0.41 & $<0.001$ \\
\hline Male & 0.60 & 0.47 to 0.73 & $<0.001$ & 0.25 & 0.10 to 0.40 & 0.002 \\
\hline Hypertension & 0.49 & 0.35 to 0.63 & $<0.001$ & 0.17 & 0.04 to 0.30 & 0.011 \\
\hline Hyperlipidaemia & 0.29 & 0.15 to 0.43 & $<0.001$ & & & \\
\hline Diabetes & 0.19 & -0.04 to 0.41 & 0.11 & & & \\
\hline Documented CAD & 0.40 & 0.16 to 0.64 & 0.001 & & & \\
\hline Family history of CAD & -0.23 & -0.37 to -0.10 & 0.001 & & & \\
\hline Ex-smoker & 0.03 & -0.12 to 0.19 & 0.68 & & & \\
\hline Current smoker & -0.15 & -0.34 to 0.03 & 0.10 & & & \\
\hline BMI $\left(\log _{2}\right)$ & 0.15 & -0.11 to 0.41 & 0.27 & 0.24 & 0.00 to 0.47 & 0.048 \\
\hline Systolic blood pressure $\left(\log _{2}\right)$ & 1.16 & 0.83 to 1.49 & $<0.001$ & 0.52 & 0.17 to 0.87 & 0.004 \\
\hline Diastolic blood pressure $\left(\log _{2}\right)$ & 0.49 & 0.16 to 0.82 & 0.004 & & & \\
\hline CT-adapted Leaman score $\left(\log _{2}\right)$ & 0.19 & 0.16 to 0.23 & $<0.001$ & 0.06 & 0.02 to 0.10 & 0.007 \\
\hline Indexed left ventricular mass $\left(\log _{2}\right)$ & 1.37 & 1.13 to 1.61 & $<0.001$ & 0.92 & 0.64 to 1.20 & $<0.001$ \\
\hline Indexed left ventricular volume $\left(\log _{2}\right)$ & 0.01 & -0.27 to 0.28 & 0.96 & & & \\
\hline Creatinine $\left(\log _{2}\right)$ & 0.41 & 0.22 to 0.61 & $<0.001$ & & & \\
\hline
\end{tabular}

${ }^{*}$ All covariates were included in the final multivariable linear regression model; only those with $p<0.05$ are presented. Full modelling is presented in online supplementary table 1.

BMI, body mass index; CAD, coronary artery disease.

multivariable adjustment. Although unexpected, it should be noted BNP has been reported as less sensitive to changes in renal function than N-terminal proBNP, while increased concentrations are typically seen in patients with advanced renal impairment that would have been ineligible for the SCOT-HEART trial. ${ }^{29}$ Indeed, there were relatively few patients in this cohort with renal impairment. Furthermore, as discussed below, there is a degree of variability in BNP assays that is driven by several factors. Small changes in serum creatinine may also be caused by factors which we did not control for, such as certain medications. Nevertheless, our findings suggest that in the context of preserved or mildly impaired renal function, changes in plasma troponin I and BNP concentrations are due to comorbid conditions rather than renal dysfunction.

Our study has several strengths. First, we applied a troponin assay with exceptional analytical characteristics, enabling us to detect circulating troponin in $99.6 \%$ of the study population and to determine troponin concentrations accurately in 96.8\%. Second, we made use of state-of-the-art CT imaging using a 320-slice scanner to quantify coronary atherosclerotic burden and left ventricular mass and volume according to previously validated techniques. Third, as this study was nested within a trial randomising allocation to CT imaging, we have minimised case ascertainment bias that may arise when

Table 3 Univariable and multivariable associations for B-type natriuretic peptide

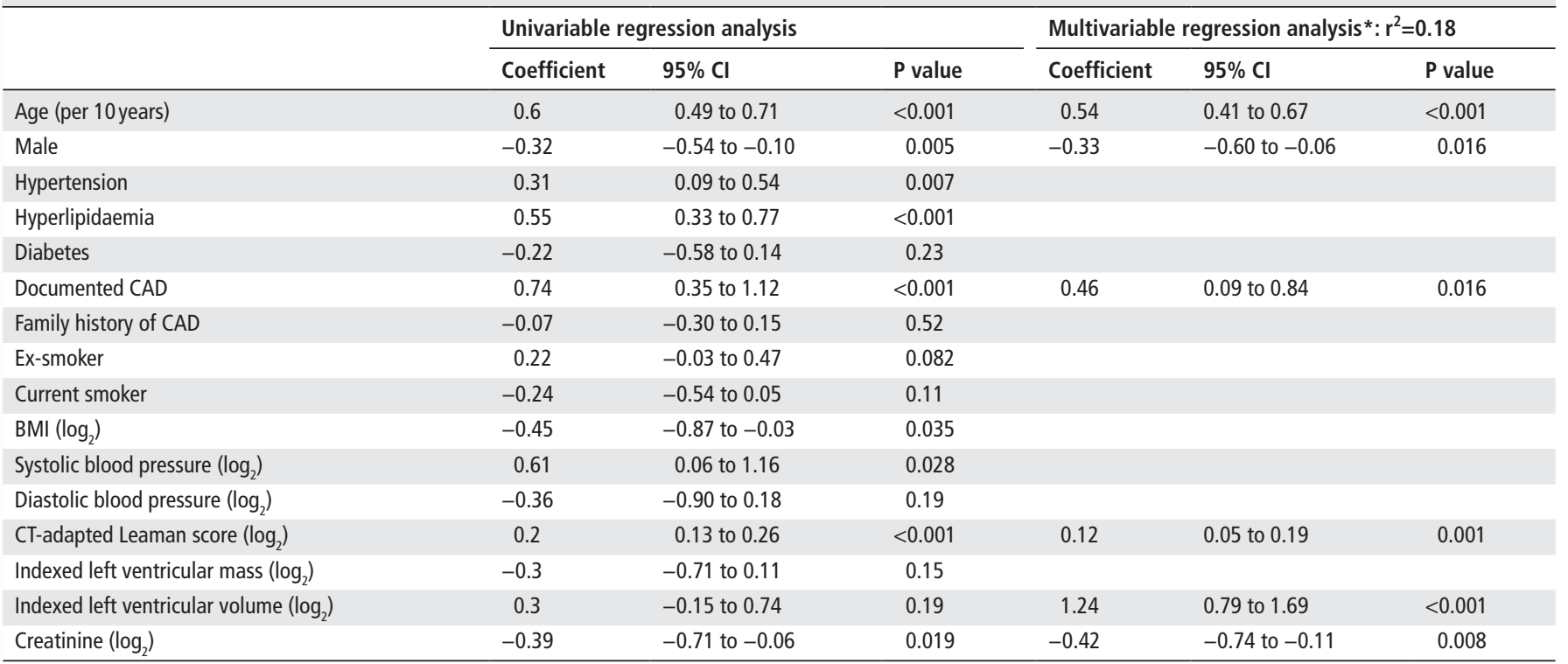

*All covariates were included in the final multivariable linear regression model; only those with $\mathrm{p}<0.05$ are presented. Full modelling is presented in online supplementary table 2.

$\mathrm{BMI}$, body mass index; $\mathrm{CAD}$, coronary artery disease. 


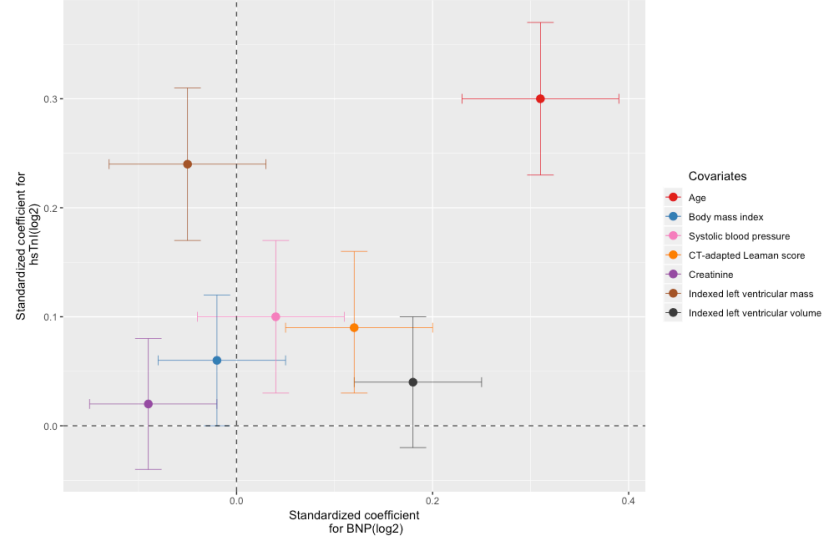

Figure 2 Multivariable logistic regression analysis for cardiac biomarkers. Multivariable logistic regression and relative contribution from selected covariates to biomarker concentrations. Log-transformed continuous covariates from the final multivariable models for highsensitivity troponin I (hsTnl) and B-type natriuretic peptide (BNP) were selected and scaled ( $(\mathrm{x}-$ mean)/SD) in order to present each variable's relative contribution to the final model. $95 \% \mathrm{Cls}$ are provided for both troponin (y axis) and BNP (x axis).

imaging decisions are dependent on clinical suspicion of coronary disease. Finally, we achieved detailed and accurate phenotypic characterisation of participants.

There are several limitations. First, key exclusion criteria for the study included age $>75$ years or severe renal impairment (estimated glomerular filtration rate $<30 \mathrm{~mL} /$ $\min / 1.73 \mathrm{~m}^{2}$ ); the determinants of biomarker concentrations in these contexts therefore remain uncertain. Nevertheless, SCOT-HEART was pragmatic in design, enrolling a cohort broadly representative of real-world populations with suspected stable angina. Second, without serial plasma samples, we cannot be certain that biomarker values reflect true baseline concentrations. However, participants were recruited in a stable outpatient setting and we excluded individuals with troponin concentrations above the 99th centile and $\mathrm{BNP}>400 \mathrm{ng} / \mathrm{L},{ }^{13}$ thereby minimising the possibility of including patients with acute myocardial injury or heart failure. Third, there is some biological, genetic and analytical variability in biomarker concentrations. Current BNP assays measure a mixture of peptides, including degradation products and proBNP, while degradation in frozen BNP samples over time is well described. These limitations are common to current assays, including $\mathrm{N}$-terminal proBNP assays. Furthermore, the clinical characteristics associated with troponin and BNP that we describe are largely consistent with existing data, supporting the validity of our analysis, while BNP degradation appears to be more relevant at higher concentrations. ${ }^{30}$ Fourth, although the assessment of left ventricular mass and volume using cardiac CT is an accepted and accurate method, mid-diastolic assessment is less widely studied. This may account for the relatively high prevalence of left ventricular hypertrophy as defined by previously published thresholds, although more than one-third of patients had a diagnosis of hypertension. Finally, despite the detailed characterisation of this cohort, substantial unexplained variance in troponin and BNP concentrations remains, highlighting the need for ongoing research to better understand the information that these assays offer.
Key messages

What is already known on this subject?

- Increased cardiac troponin and B-type natriuretic (BNP) concentrations are associated with greater risk of cardiovascular events in individuals with and without established cardiac disease.

- Plasma concentrations in stable outpatients may be attributable to pathophysiological processes including hypertension, coronary atherosclerosis and left ventricular dysfunction, but clinical determinants are not fully characterised.

\section{What might this study add?}

- We demonstrate the association between overall plaque burden as assessed by CT coronary angiography (CTCA) and troponin and BNP concentrations.

- Importantly, we describe sex differences and contrasting associations with left ventricular mass and volume.

- We also demonstrate the ability of prospectively gated CTCA performed primarily for coronary artery assessment to provide additional information regarding ventricular mass and volume.

How might this impact on clinical practice?

- Although plasma troponin and BNP have prognostic value in stable patients, an understanding of their determinants-and specifically, modifiable determinants-is necessary to add clinical utility.

- With this knowledge, these plasma biomarkers have the potential to act as simple surrogate markers of treatment efficacy for targeted disease-modifying therapies.

\section{CONCLUSIONS}

Detectable plasma high-sensitivity cardiac troponin I and BNP concentrations are present in almost all patients with suspected stable coronary artery disease. These biomarkers are associated with atherosclerotic burden, but measures of underlying left ventricular structure are associated with each biomarker differentially. Cardiac biomarkers provide information regarding patient-specific modifiable targets for therapy, allowing personalisation of treatment that may further help reduce cardiovascular risk.

Acknowledgements Singulex provided reagents, calibrators and controls without charge and undertook the analysis of cardiac biomarkers.

Contributors RB and PDA drafted the manuscript. JH, AH, MCW and AM contributed to image analysis and data collection. RB, ASVS, DM and PDA contributed to data analysis. MRD, DEN and NLM contributed to the study conceptualisation, methodology, oversight and funding acquisition. All authors contributed to the final manuscript.

Funding The Chief Scientist Office of the Scottish Government (CZH/4/588) funded the SCOT-HEART trial with supplementary support from the British Heart Foundation (RE/13/3/30183), Edinburgh and Lothians Health Foundation Trust, and the Heart Diseases Research Fund. DEN (CH/09/002, RE/18/5/34216), NLM (FS/16/14/32023) and MRD (FS/14/78/31020) are supported by the British Heart Foundation. DEN is also the recipient of a Wellcome Trust Senior Investigator Award (WT103782AIA). MCW is supported by the Chief Scientist Office of the Scottish Government Health and Social Care Directorates (PCL/17/04). DM is supported by an Intermediate Clinical Fellowship and Beit Fellowship from the Wellcome Trust (201492/Z/16/Z).

Competing interests None declared.

Patient consent for publication Not required.

Ethics approval Ethical approval was provided by the South East Scotland Research Ethics Committee 02, Edinburgh, UK (10/S1102/43). 
Provenance and peer review Not commissioned; externally peer reviewed.

Open access This is an open access article distributed in accordance with the Creative Commons Attribution 4.0 Unported (CC BY 4.0) license, which permits others to copy, redistribute, remix, transform and build upon this work for any purpose, provided the original work is properly cited, a link to the licence is given, and indication of whether changes were made. See: https://creativecommons.org/ licenses/by/4.0/

\section{ORCID iDs}

Rong Bing http://orcid.org/0000-0002-8305-4906

Michelle C Williams http://orcid.org/0000-0003-3556-2428

David A McAllister http://orcid.org/0000-0003-3550-1764

Nicholas L Mills http://orcid.org/0000-0003-1926-5925

Philip D Adamson http://orcid.org/0000-0002-6177-956X

\section{REFERENCES}

1 Zeller T, Tunstall-Pedoe H, Saarela O, et al. High population prevalence of cardiac troponin I measured by a high-sensitivity assay and cardiovascular risk estimation: the MORGAM Biomarker Project Scottish Cohort. Eur Heart J 2014;35:271-81.

2 Ford I, Shah AS, Zhang R, et al. High-Sensitivity Cardiac Troponin, Statin Therapy, and Risk of Coronary Heart Disease. J Am Coll Cardiol 2016;68:2719-28.

3 Redfield MM, Rodeheffer RJ, Jacobsen SJ, et al. Plasma brain natriuretic peptide concentration: impact of age and gender. J Am Coll Cardiol 2002;40:976-82.

4 Azevedo A, Bettencourt P, Barros H. Demographic, clinical and echocardiographic determinants of B-type natriuretic peptide plasma concentration. A population-based study. Rev Port Cardiol 2007;26:105-13.

5 de Lemos JA, Drazner MH, Omland T, et al. Association of troponin T detected with a highly sensitive assay and cardiac structure and mortality risk in the general population. JAMA 2010:304:2503-12.

6 McEvoy JW, Chen Y, Nambi V, et al. High-Sensitivity Cardiac Troponin T and Risk of Hypertension. Circulation 2015;132:825-33.

7 Yancy CW, Jessup M, Bozkurt B, et al. 2017 ACC/AHA/HFSA Focused Update of the 2013 ACCF/AHA Guideline for the Management of Heart Failure: A Report of the American College of Cardiology/American Heart Association Task Force on Clinical Practice Guidelines and the Heart Failure Society of America. J Card Fail 20172017;23:628-51

8 Eggers KM, Lind L, Ahlström H, et al. Prevalence and pathophysiological mechanisms of elevated cardiac troponin I levels in a population-based sample of elderly subjects. Eur Heart J 2008:29:2252-8.

9 Goetze JP, Mogelvang R, Maage L, et al. Plasma pro-B-type natriuretic peptide in the general population: screening for left ventricular hypertrophy and systolic dysfunction. Eur Heart J 2006;27:3004-10.

10 SCOT-HEART investigators. CT coronary angiography in patients with suspected angina due to coronary heart disease (SCOT-HEART): an open-label, parallel-group, multicentre trial. Lancet 2015;385:2383-91.

11 Newby DE, Adamson PD, Berry C, et al. Coronary CT Angiography and 5-Year Risk of Myocardial Infarction. N Engl J Med 2018;379:924-33.

12 Newby DE, Williams MC, Flapan AD, et al. Role of multidetector computed tomography in the diagnosis and management of patients attending the rapid access chest pain clinic, The Scottish computed tomography of the heart (SCOT-HEART) trial: study protocol for randomized controlled trial. Trials 2012;13:184.

13 Maisel A, Mueller C, Adams K, et al. State of the art: using natriuretic peptide levels in clinical practice. Eur J Heart Fail 2008:10:824-39.

14 Andreini D, Pontone G, Mushtaq S, et al. Long-term prognostic impact of CTLeaman score in patients with non-obstructive CAD: Results from the COronary CT Angiography EvaluatioN For Clinical Outcomes InteRnational Multicenter (CONFIRM) study. Int J Cardiol 2017;231:18-25.

15 Klein R, Ametepe ES, Yam Y, et al. Cardiac CT assessment of left ventricular mass in mid-diastasis and its prognostic value. Eur Heart J Cardiovasc Imaging 2017:18:95-102.

16 Boczar KE, Alam M, Chow BJ, et al. Incremental prognostic value of estimated LV enddiastolic volume by cardiac CT. JACC Cardiovasc Imaging 2014;7:1280-1.

17 Fuchs A, Mejdahl MR, Kühl JT, et al. Normal values of left ventricular mass and cardiac chamber volumes assessed by 320-detector computed tomography angiography in the Copenhagen General Population Study. Eur Heart J Cardiovasc Imaging 2016:17:1009-17.

18 Juneau D, Erthal F, Clarkin 0, et al. Mid-diastolic left ventricular volume and mass: Normal values for coronary computed tomography angiography. J Cardiovasc Comput Tomogr 2017;11:135-40.

19 Walker JR, Abadi S, Solomonica A, et al. Left-sided cardiac chamber evaluation using single-phase mid-diastolic coronary computed tomography angiography: derivation of normal values and comparison with conventional end-diastolic and end-systolic phases. Eur Radiol 2016;26:3626-34

20 Ah W, Estis J, Helestine P, et al. High-sensitivity cardiac troponin I in a large community-based population at risk for cardiovascular disease. Clin Chem 2015;61(S10):S121

21 Apple FS, Simpson PA, Murakami MM. Defining the serum 99th percentile in a normal reference population measured by a high-sensitivity cardiac troponin I assay. Clin Biochem 2010;43:1034-6.

22 Rubin DB. Multiple Imputation for Nonresponse in Surveys. New York: John Wiley \& Sons Inc, 1987.

23 Ridker PM, Everett BM, Thuren T, et al. Antiinflammatory Therapy with Canakinumab for Atherosclerotic Disease. N Eng/ J Med 2017;377:1119-31.

24 Adamson PD, Hunter A, Madsen DM, et al. High-Sensitivity Cardiac Troponin I and the Diagnosis of Coronary Artery Disease in Patients With Suspected Angina Pectoris. Circ Cardiovasc Qual Outcomes 2018:11:e004227.

25 Chin CW, Shah AS, McAllister DA, et al. High-sensitivity troponin I concentrations are a marker of an advanced hypertrophic response and adverse outcomes in patients with aortic stenosis. Eur Heart J 2014;35:2312-21.

26 Zhao Z, Wang H, Jessup JA, et al. Role of estrogen in diastolic dysfunction. Am J Physiol Heart Circ Physiol 2014;306:H628-H640.

27 Bachmann KN, Huang S, Lee H, et al. Effect of Testosterone on Natriuretic Peptide Levels. J Am Coll Cardiol 2019;73:1288-96.

28 Lamb EJ, Kenny C, Abbas NA, et al. Cardiac troponin I concentration is commonly increased in nondialysis patients with CKD: experience with a sensitive assay. Am J Kidney Dis 2007;49:507-16.

29 Srisawasdi P. Vanavanan S, Charoenpanichkit C, et al. The effect of renal dysfunction on BNP, NT-proBNP, and their ratio. Am I Clin Pathol 2010;133:14-23.

30 Pereira M, Azevedo A, Severo M, et al. Long-term stability of endogenous B-type natriuretic peptide after storage at $-20^{\circ} \mathrm{C}$ or $-80^{\circ} \mathrm{C}$. Clinical chemistry and laboratory medicine 2008:p. 1171 